\title{
SIGNIFICANT QUALITY OF FRAGMENTED FORESTS IN OIL PALM PLANTATIONS: LESSON FROM THE ASSEMBLAGE STRUCTURE OF FROGS (Amphibia: Anura)
}

\author{
RAMLAH ZAINUDIN*; ELVY QUATRIN DEKA*; MUHAMMAD FADZIL AMRAM*; SHARIZATTY MOHD \\ RAIS*; NUR AMIRAH SUNGIF*; MARLY MATLEEN AUGUSTINE AGOH*; NOORAINA ATIRA ALAUDIN*; \\ MOHAMAD AMIRUL AZMI* and JAYASILAN MOHD-AZLAN ${ }^{\star}$
}

\begin{abstract}
Being known as a good environmental indicator, the anuran is an ideal animal model for investigating the quality of High Conservation Value (HCV) areas (fragmented forests) in maintaining or enhancing biodiversity values in an oil palm plantation. Using non-metric multidimensional scaling (NMDS) frogs from forest fragments and oil palm were investigated and compared, to identify species assemblages and guild. Our findings showed that species diversity differed significantly between High Conservation Values and the plantation areas. The High Conservation Values showed highest percentage of species endemism, and species of conservation importance in the areas. The NMDS analysis further suggests that the anuran assemblages at the oil palm plantation were disjunctive as the HCV areas provide isolated assemblages to the anuran species, different from that of the plantations. The number of species was found to be significantly influenced by water temperature, turbidity, salinity, and level of dissolved oxygen. Overall, the HCV areas supports high species diversity, including endemic and near threatened species compared to the plantation areas. Hence, these HCV areas are functioning and should be maintained as high priority areas for faunal conservation in an oil palm plantation.
\end{abstract}

Keywords: frog assemblages, HCV areas, oil palm plantation, endemic species, environmental indicator.

Date received: 30 August 2016; Sent for revision: 22 March 2017; Received in final form: 30 July 2018 ; Accepted: 14 October 2019.

\section{INTRODUCTION}

Bornean amphibians are higly endemic that occupies a wide variety of habitats, from lowland dipterocarp forest to sub-montane forest. They are very sensitive to their environment and water quality in particular and thus, can serve as good environmental indicators. Negative changes in water quality have known to cause a higher rate of tadpole fatality and

Department of Zoology, Faculty of Resource Science and Technology, Universiti Malaysia Sarawak, 94300 Kota Samarahan, Sarawak, Malaysia.

E-mail: zramlah@unimas.my deformation. Berry et al. (2010) revealed that logging has adverse effect on amphibian's abundance. Thus, the anuran is an ideal model for investigating the effectiveness of High Conservation Value (HCV) areas in maintaining or enhancing biodiversity values.

HCV areas refer to forest areas with high ecological or social values that need to be maintained or enhanced (Jennings et al., 2003; Sanath, 2008). Specifically, according to Jennings et al. (2003), HCV areas are forests that have significant concentrations of biodiversity values, and contain viable populations of species existence, as well as rare, threatened or endangered ecosystems. 
Additionally, these areas contain significantly large landscape-level forests and management unit, and provide basic services of nature in critical situations (e.g. watershed protection, erosion control). Finally, $\mathrm{HCV}$ areas are deemed fundamental in providing the basic needs of local communities. Most of the bigger plantations must comply with the regulation of having several HCV areas in their large scale plantations so that they can provide areas and corridors for wildlife refugia that may have once inhabited pristine and undisturbed forests.

The impact of rainforest degradation due to its conversion to oil palm plantations on amphibians is relatively unknown. Additionally, information on anuran communities in oil palm plantations is still limited with only three studies to date (Gillespie et al., 2012; Faruk et al., 2013; Norhayati et al., 2014).

Faruk et al. (2013) found that anuran communities in plantations consisted of species that thrive in disturbed areas. Furthermore, Harrison and Bruna (1999) stated that fragmented habitats cannot simply represent the original habitat and that their biotas may be altered to a great extent, thus assuming that conserving small remnant areas will not guarantee to preserve species successfully. Recently, Kurz et al. (2016) found 719 frogs from 14 species across eighteen 2.25-ha oil palm plots. Frog richness was $31 \%$ lower in replanted oil palm (nine species) than mature oil palm (13 species). They concluded that management decisions within tropical agricultural landscapes also have a profound impact on biodiversity. Furthermore, Norhayati et al. (2014) revealed that the remediation diversity in the green corridor (HCV) of oil palm plantation in Felda Pasoh 3 is yet to be achieved with amphibian species tend to avoid overlapping of microhabitat based on species guild. Thus, they suggested that the green corridor should be widened to allow space for foraging and territorial defence, planted not only with trees but also shrubs and herbs to improve the ground cover for the amphibians and also to include other animal groups, such as birds and mammals.

Anurans are susceptible to habitat change, thus, their community ecology would be highly affected if the areas are constantly modified and altered by human disturbances. With previous findings as mentioned above as well as the sensitivity of the anurans, it is important to assess connectivity of the anuran assemblages at HCV areas. Thus, the study aims to compare species diversity, assemblage stucture; and also determine the effects of water quality on species assemblage and abundances along the established HCV areas in an oil palm plantation. It is hoped that this study will provide insight into the biodiversity value of maintaining $\mathrm{HCV}$ areas thus validated their function as areas of biodiversity maintenance in huge oil palm plantations.

\section{MATERIALS AND METHODS}

Field samplings were conducted on three separate occasions between September 2013 and March 2015 at Saremas and Sagermas Oil Palm Plantations in Miri, Sarawak, Malaysia (Figure 1). These oil palm areas were established in 2008 with a total size of 777.90 ha for Saremas 1 and 2, and approximately 474.88 ha for Sagermas plantation. Various riparian sites representing fragmented forest $(\mathrm{HCV})$ and plantation areas were chosen (Figures $1 a$ and $1 b$ ).

Several streams of HCV areas consisting of Hulu Sungai Bukit Durang (N0327.430', E11349.933), Sungai Kolam (N03 $\left.29.585^{\prime}, \mathrm{E}^{\circ} 13^{\circ} 49.980^{\prime}\right)$, Sungai Akuarium (N0141.515', E109 $\left.{ }^{\circ} 50.843^{\prime}\right)$, Hulu Sungai Batu Anchau (N03 $30.553^{\prime}$, E113 $\left.{ }^{\circ} 48.942^{\prime}\right)$

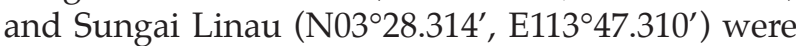
categorised as fragmented forests (HCV areas by Sanath, 2008), while Hilir Sungai Bukit Durang $\left(\mathrm{N} 03^{\circ} 27.188^{\prime}, \mathrm{E}^{\circ} 13^{\circ} 50.273^{\prime}\right)$, Tengah Sungai Batu Anchau (N03 $30.553^{\prime}$, E113 $\left.^{\circ} 48.942^{\prime}\right)$ and Hilir Sungai Batu Anchau were categorised as plantation areas. Water quality assessments such as $\mathrm{pH}$, temperature, dissolved oxygen and salinity were assessed using Eutech water quality instrument, and HANNA instruments while turbidity with Thermo Scientific Orion. Assessments were done with three replicates for each site.

Line transects of 1-2 $\mathrm{km}$ distance (depending on the length of the HCV forest) with $5 \mathrm{~m}$ both sides (left and right) were employed along the stream banks and plantation areas. Visual Encounter Survey (VES) and frog sounds were used to detect the anurans along $5 \mathrm{~m}$ buffer parrarel to the rivers. The animals were located by headlamps and caught by hand. All specimens were preserved in $10 \%$ formalin and later stored in $70 \%$ alcohol as vouchers deposited at the UNIMAS Zoological Museum. The specimens were identified based on the characteristics described by Inger and Stuebing (2005).

Species diversity, evenness and richness indices of Shannon, Simpson and Margalef were calculated. A non-metric multidimensional scaling (NMDS) was employed to identify pattern of frog assemblages within study sites and further determine the species guild. Spearman's rs and Kendall correlations were used to analyse the effect of water quality to anuran species abundance within study sites. All analyses were also conducted using Paleontological Statistics (PAST) version 2.14 (Hammer et al., 2001).

\section{RESULTS AND DISCUSSION}

A total of 181 individuals representing seven families, 12 genera and 24 species were obtained from eight sampling sites (Table 1). From this, $40 \%$ was represented by the family Dicroglossidae, $28 \%$ from Ranidae, 10\% from Bufonidae, 12\% from 


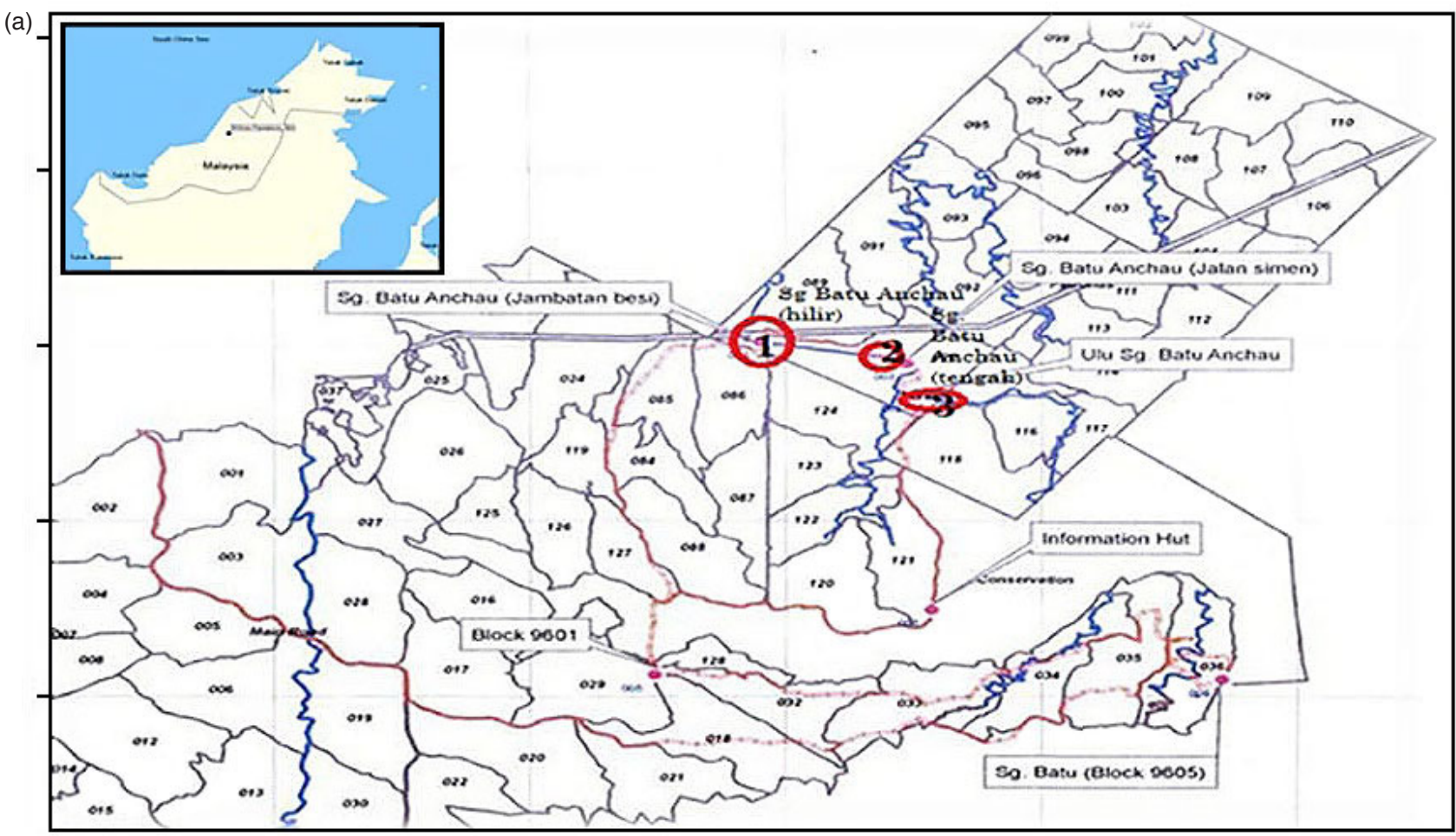

(b) Reference IIs:

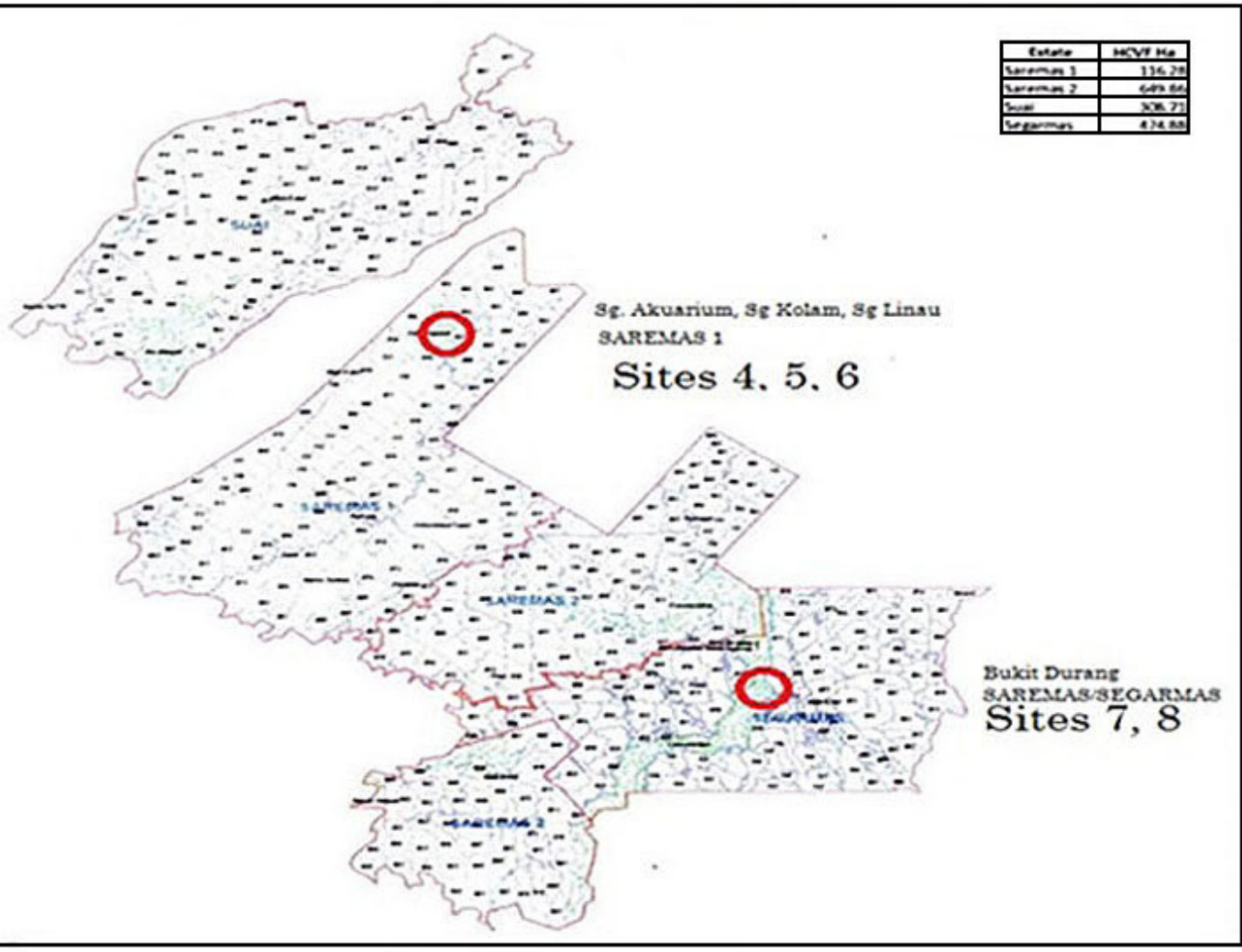

Figure 1. Site localities of frog sampling at the Saremas (a) and Sagermas Oil Palm Plantations (b). Top left: map of Sarawak (Malaysian Borneo) showing study localities at Wilmar Oil Palm Plantations (Saremas and Sagermas plantations).

Megophyridae, 5\% represented by Rhacophoridae, $3 \%$ from Ceratobatrachidae and $2 \%$ from Microhylidae. The most common species was Limnonectes kuhlii followed by Pulchrana glandulosa (11.60\%, Appendix 1), H. raniceps $(8.29 \%), H$. signata $(8.29 \%)$ and L. leporinus $(8.29 \%)$. In contrast, Alcalus sariba formerly known as Alcalus shelfodi (Appendix 1), Pelophyrne signata, L. malasienus, Leptorachium sp. and $P$. baramica were represented by single individual (Table 1).

Diversity indices of Shannon, Simpson and Margalef were found to be the highest at Sungai 
Akuarium (Table 2). With the exception of Sg Kolam (mostly surrounded by plantations), all the $\mathrm{HCV}$ areas showed the highest diversity ranging from 1.818-2.433 (Shannon), 0.8166-0.8840 (Simpson) and 2.339-3.71 (Margalef) as compared to the plantation areas with diversity indices of 1.099-1.332 (Shannon), 0.6667 to 0.72 (Simpson) and 1.16-1.874 (Margalef). Shannon diversity t-test within study sites significantly supports the difference between HCV species diversity to that of plantation areas (Table 3 ), thus, suggesting that species richness is lower in oil palm plantations than in secondary forests in Borneo.

$\mathrm{HCV}$ areas in oil palm plantations are considered to be of critical importance if the areas are occupied by threatened and endangered species, as well as endemic species (Jennings et al., 2003). This study revealed that most of the HCV areas in Saremas and Sagermas Oil Palm Plantations are critically important as the areas were inhabited by near threatened and endemic species to Borneo (high endemism at Sg Akuarium, Table 4). Thus, HCV areas in these plantations function as a refugium for the remaining species from a larger subset of species before the landscape change and forest fragmentation.

NMDS, a unique pattern of frog assemblages were identified within the study sites as in Figure 2. Component loadings may be considered a measure of relative importance of each variable in the extracted NMDS final coordinate axis. For example, variables Sg Akuarium (0.3698) and Ulu Sg Batu Anchau (0.5283) have high positive loadings on the dimension 1 axis, suggesting anuran species prefer these two $\mathrm{HCV}$ areas for their assemblage sites.

TABLE 1. RELATIVE ABUNDANCE OF FROG SPECIES AT WILMAR OIL PALM PLANTATIONS

\begin{tabular}{llrc}
\hline Family & Species & N & Relative abundance \\
\hline Ceratobatrachidae & Alcalus baluensis & 4 & 2.21 \\
& Alcalus sariba & 1 & 0.55 \\
Bufonidae & Ansonia leptopus & 3 & 1.66 \\
& Ansonia longidigita & 2 & 1.10 \\
& Ingerophrynus divergens & 13 & 7.18 \\
Dicroglossidae & Pelophyrne signata & 1 & 0.55 \\
& Limnonectes finchi & 4 & 2.21 \\
& Limnonectes ingeri & 12 & 6.63 \\
& Limnonectes kuhlii* & 31 & 17.13 \\
& Limnonectes leporinus & 15 & 8.29 \\
& Limnonectes malasienus & 1 & 0.55 \\
Megophyridae & Limnonectes paramacrodon & 4 & 2.21 \\
& Fejervarya limnocharis & 6 & 3.32 \\
& Leptobrachella mjobergi & 9 & 4.97 \\
& Leptobrachium sp. & 1 & 0.55 \\
Microhylidae & Leptobrachium abotti & 4 & 2.21 \\
Ranidae & Megophyrs nasuta & 6 & 3.32 \\
& Metaphrynella sundana & 4 & 2.21 \\
& Pulchrana baramica & 1 & 0.55 \\
& Pulchrana glandulosa & 21 & 11.60 \\
Rhacophoridae & Chalcorana raniceps & 15 & 8.29 \\
& Pulchrana signata & 15 & 8.29 \\
& Polypedates leucomystax & 6 & 3.32 \\
& Nyctixalus pictus & 2 & 1.10 \\
\hline
\end{tabular}

Note: *Denotes the most abundance species in Wilmar Oil Palm Plantations.

TABLE 2. DIVERSITY INDICES AMONG STUDY LOCALITIES AT WILMAR OIL PALM PLANTATIONS

\begin{tabular}{llccc}
\hline \multicolumn{1}{c}{ Location } & Site & Shannon_H & Simpson & Margalef \\
\hline Hulu Sg Bukit Durang & HCV & 2.018 & 0.8364 & 2.511 \\
Hilir Sg Bukit Durang & Plantation & 1.332 & 0.7200 & 1.864 \\
Sg Kolam & HCV & 1.011 & 0.6111 & 1.116 \\
Sg Akuarium* & HCV & 2.433 & 0.8840 & 3.71 \\
Hilir Sg Batu Anchau & Plantation & 1.311 & 0.7160 & 1.365 \\
Tengah Sg Batu Anchau & Plantation & 1.099 & 0.6667 & 1.116 \\
Ulu Sg Batu Anchau & HCV & 2.016 & 0.8530 & 2.056 \\
Sg Linau & HCV & 1.818 & 0.8166 & 2.339 \\
\hline
\end{tabular}

Note: * Denoted the area that has the highest diversity indices.

HCV - High Conservation Value. 


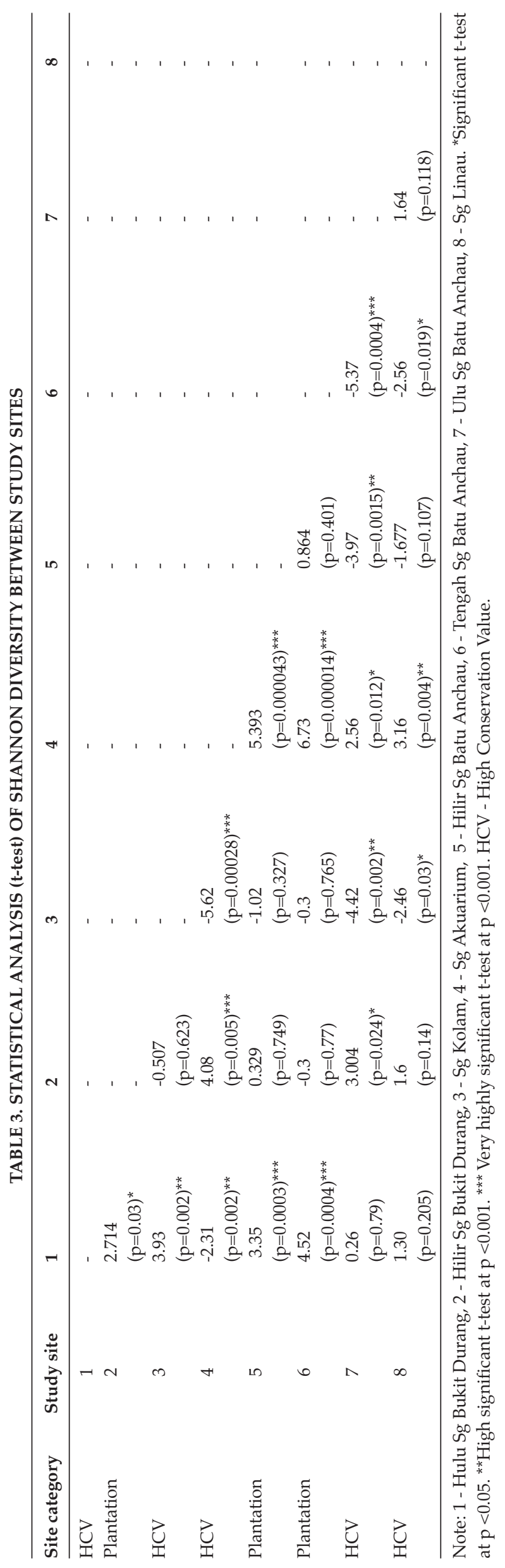

High negative loadings on the dimension 1 [Hilir Sg Bt Anchau (-0.175)] proved that the anuran species do not prefer to assemble at the plantation areas while negative loading of dimesion 2 axis at Hulu Sg Bukit Durang (-0.4196) might suggests of forest conversion as this site is within the $\mathrm{HCV}$ areas.

The findings above suggest that the anuran assemblages at the oil palm plantation were disjunctives, in such a way that the $\mathrm{HCV}$ areas provide isolated assemblages to the anuran species differ from that of the plantations. However, two $\mathrm{HCV}$ areas of Sg Kolam amd Sg Linau were grouped together in the plantation guild probably due to their locations at the forest edge and surrounded by plantation areas. Another possible explanation for the disjunctive of anuran assemblages is the environmental condition of the study sites. Table 5 shows water quality assessment within the study sites together with the number of frog species caught. Mann-Whitney pairwise comparisons revealed significant differences only between Tengah Sg Batu Anchau (plantation) and the following HCV areas: Sg Kolam (HCV area edge), Hulu Sg Bukit Durang (HCV area), Ulu Sg Batu Anchau (HCV area) and Sg Linau (HCV area edge) as in Table 6. Moreover, both Spearman's rs and Kendall correlations showed that the number of species found was significantly influenced by turbidity, salinity, temperature and dissolve oxygen (DO) (Tables 7 and 8).

Ecological groups among individuals defined by NMDS for the entire assemblages of anurans at $\mathrm{HCV}$ areas and plantations are shown in Figure 3. Four ecological guilds based on Euclidean distance of species assemblages are as follows: i) plantation assemblages (Hilir Sg Bukit Durang, Sg Kolam, Hilir Sg Batu Anchau, Tengah Sg Batu Anchau, and Sg Linau); ii) HCV1 areas assemblages (Hulu Sg Bukit Durang); iii) HCV2 assemblages (Sg Akuarium) and iv) HCV 3 areas assemblages (Ulu Sg Batu Anchau). A minimum spanning tree would be one with the lowest connection revealing the longest pathway connection of the three HCV areas guilds with the plantation guild.

Low anuran species richness in oil palm plantations as compared in the HCV forested areas is consistent with previous studies that suggest oil palm plantations are dominated by disturbancetolerant species (Kurz et al., 2016; Sheridan, 2012; Wu et al., 2006; Gillespie et al., 2012). Lack of food sources may also contribute to the low number of species. The utilisation of pesticides may have limited the prey availability. For example, the ant populations in oil palm plantations were found to be less diverse as compared to the forested areas where it is known to support only $5 \%$ of ant species than the forested region (Bruehl and Eltz, 2010; Fayle et al., 2010; 2013; Lucey et al., 2014). In addition, Liow et al. (2001) also found that oil palm plantations in Malaysia have less number of bee species. 


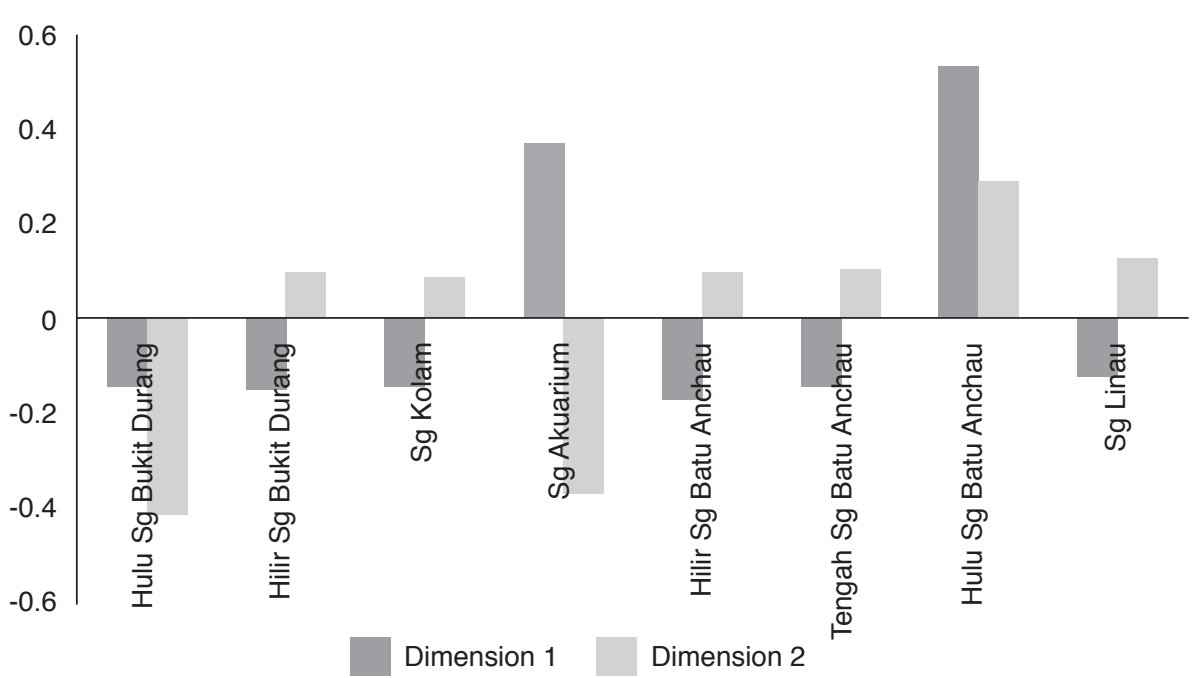

Figure 2. Final Coordinate Dimension (FDC) 1 and 2 of non-metric multidimensimal scaling (NMDS) (PROXSCAL) on species assemblages at Saremas and Sagermas plantations.

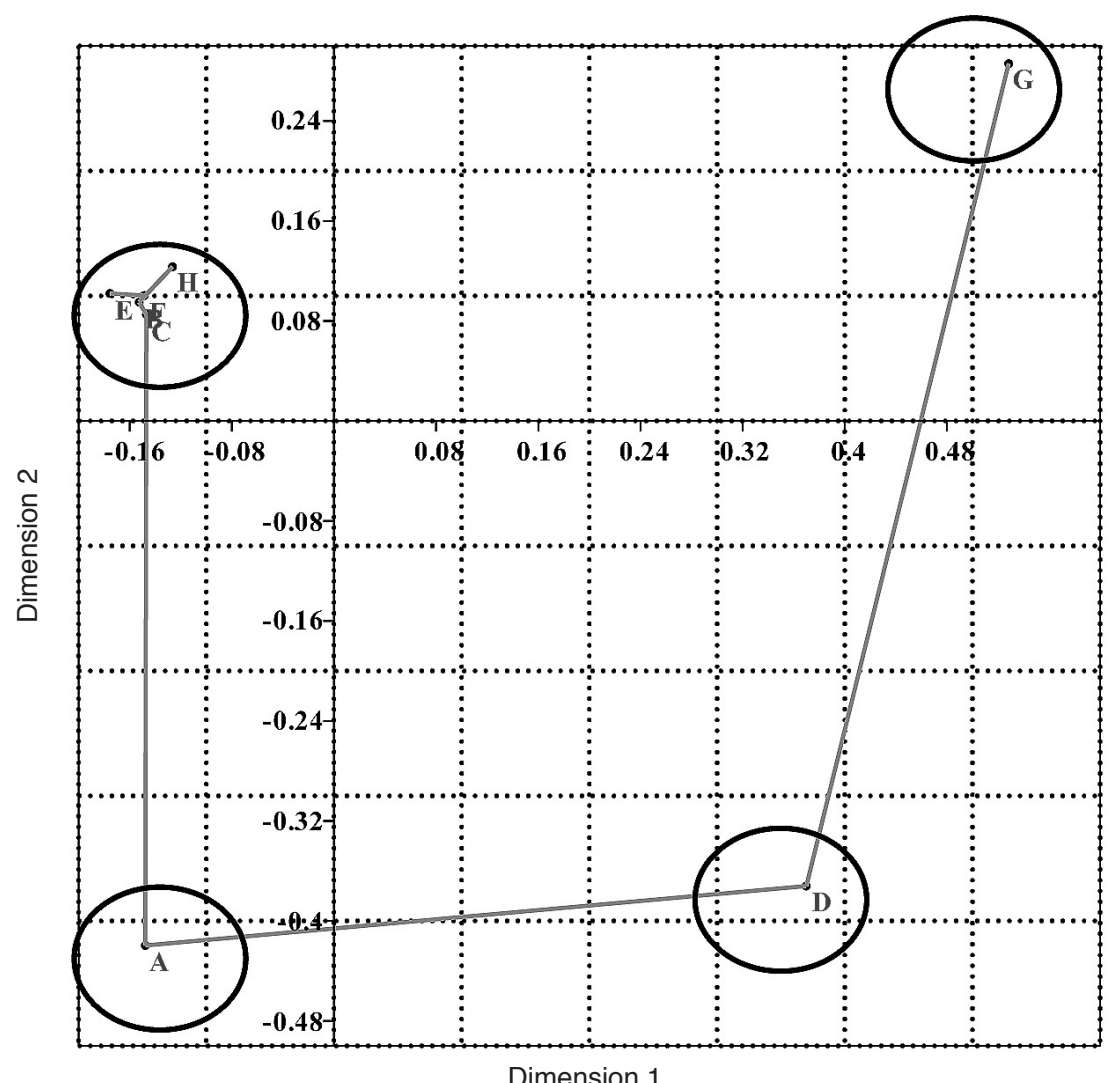

Note: A - Hulu Sg Bukit Durang, B - Hilir Sg Bukit Durang, C - Sg Kolam, D - Sg Akuarium, E - Hilir Sg Batu Anchau, F -Tengah Sg Batu Anchau, G - Ulu Sg Batu Anchau, H - Sg Linau. E, F and B are plantations. A, C, D, G, H are High Conservation Value (HCV) areas.

Figure 3. Non-metric multidimensional scaling (NMDS) scatter plot of minimum spanning network based on Similarity Index of Euclidean on anuran species assemblages at Wilmar Oil Palm Plantations.

Faruk et al. (2013) and Froster et al. (2011) stated that the canopy layer of plantation areas are much lower and open compared to forest areas which leads to drier conditions. Not all frogs can tolerate extreme conditions especially the Microhylids (narrowmouthed frog) that have the ability to secrete thick and sticky mucus from their glands (Inger and Stuebing, 2005). These frogs need moisture to control their excessive mucus secretion and yet the moisture can only be found and maintained at the $\mathrm{HCV}$ forested regions due to higher canopy cover. Besides, most of the forest frogs (Megophridae, some Bufonids, Ranids, Rhacophorids) are specifically adapted to the forest environment as some frogs needs leaf litter, while others perch on shrubs and herbaceous plants, or live in tree holes or buttresses. 
TABLE 4. ENDEMIC SPECIES AND IUCN STATUS OF THE ANURANS AT WILMAR

(Saremas and Sagermas) OIL PALM PLANTATIONS

\begin{tabular}{|c|c|c|c|c|c|c|}
\hline Locality & $\begin{array}{c}\text { Site } \\
\text { category }\end{array}$ & Family & Species & $\begin{array}{c}\text { No. of } \\
\text { individual }\end{array}$ & IUCN 2016 & $\begin{array}{l}\text { Percent of } \\
\text { endemism }\end{array}$ \\
\hline $\begin{array}{l}\text { Hulu } \\
\text { Sg Bukit Durang } \\
(\text { N species }=10)\end{array}$ & HCVF & $\begin{array}{l}\text { Bufonidae } \\
\text { Bufonidae } \\
\text { Rhacophoridae } \\
\text { Ranidae } \\
\text { Dicroglossidae } \\
\text { Dicroglossidae } \\
\text { Megophyridae } \\
\text { Megophyridae } \\
\text { Ranidae } \\
\text { Bufonidae }\end{array}$ & $\begin{array}{l}\text { Ingerophrynus divergens* } \\
\text { Ansonia leptopus } \\
\text { Polypedates leucomystax } \\
\text { Pulchrana signata } \\
\text { Limnonectes kuhlii } \\
\text { Limnonectes ingeri* } \\
\text { Leptobrachium abbotti* } \\
\text { Leptobrachium sp. } \\
\text { Pulchrana glandulosa } \\
\text { Pelophryne signata }\end{array}$ & $\begin{array}{l}9 \\
3 \\
3 \\
3 \\
9 \\
1 \\
4 \\
1 \\
2 \\
1\end{array}$ & $\begin{array}{l}\text { Least concern (LC) } \\
\text { Near threatened (NT) } \\
\text { LC } \\
\text { LC } \\
\text { LC } \\
\text { NT } \\
\text { LC } \\
\text { Not listed } \\
\text { LC } \\
\text { Near threatened }\end{array}$ & 30 \\
\hline $\begin{array}{l}\text { Hilir } \\
\text { Sg Bukit Durang } \\
(\text { N species }=4)\end{array}$ & Plantation & $\begin{array}{l}\text { Rhacophoridae } \\
\text { Ranidae } \\
\text { Ranidae } \\
\text { Dicroglossidae }\end{array}$ & $\begin{array}{l}\text { Polypedates leucomystax } \\
\text { Pulchrana glandulosa } \\
\text { Chalcorana raniceps } \\
\text { Limnonectes paramacrodon }\end{array}$ & $\begin{array}{l}1 \\
2 \\
1 \\
1\end{array}$ & $\begin{array}{l}\text { LC } \\
\text { LC } \\
\text { LC } \\
\text { NT }\end{array}$ & 0 \\
\hline $\begin{array}{l}\text { Sg Kolam } \\
(\mathrm{N} \text { species = 3) }\end{array}$ & HCVF & $\begin{array}{l}\text { Dicroglossidae } \\
\text { Megophyridae } \\
\text { Ceratobatrachidae }\end{array}$ & $\begin{array}{l}\text { Limnonectes kuhlii } \\
\text { Leptobrachella mjobergi* } \\
\text { Alcalus baluensis }\end{array}$ & $\begin{array}{l}3 \\
2 \\
1\end{array}$ & $\begin{array}{l}\mathrm{LC} \\
\mathrm{LC} \\
\mathrm{LC}\end{array}$ & 33.3 \\
\hline $\begin{array}{l}\text { Sg Akuarium } \\
(\text { N species = 16) }\end{array}$ & HCVF & $\begin{array}{l}\text { Dicroglossidae } \\
\text { Dicroglossidae } \\
\text { Ranidae } \\
\text { Megophyridae } \\
\text { Bufonidae } \\
\text { Ranidae } \\
\text { Dicroglossidae } \\
\text { Ranidae } \\
\text { Megophyridae } \\
\text { Bufonidae } \\
\text { Dicroglossidae } \\
\text { Dicroglossidae } \\
\text { Dicroglossidae } \\
\text { Microhylidae } \\
\text { Ranidae } \\
\text { Dicroglossidae }\end{array}$ & $\begin{array}{l}\text { Limnonectes kuhlii } \\
\text { Limnonectes leporinus* } \\
\text { Pulchrana glandulosa } \\
\text { Megophyrs nasuta } \\
\text { Ingerophrynus divergens* } \\
\text { Pulchrana signata } \\
\text { Limnonectes ingeri* }^{*} \\
\text { Chalcorana raniceps } \\
\text { Leptobracella mjobergi* } \\
\text { Ansonia longidigita* } \\
\text { Limnonectes paramacrodon } \\
\text { Limnonectes finchi* } \\
\text { Limnonectes malesianus } \\
\text { Metaprynella sundana } \\
\text { Pulchrana baramica } \\
\text { Fejervarya limnocharis }\end{array}$ & $\begin{array}{r}14 \\
6 \\
5 \\
2 \\
4 \\
5 \\
1 \\
3 \\
7 \\
2 \\
2 \\
2 \\
1 \\
1 \\
1 \\
1\end{array}$ & $\begin{array}{l}\text { LC } \\
\text { LC } \\
\text { LC } \\
\text { LC } \\
\text { LC } \\
\text { LC } \\
\text { NT } \\
\text { LC } \\
\text { LC } \\
\text { NT } \\
\text { NT } \\
\text { LC } \\
\text { NT } \\
\text { LC } \\
\text { LC } \\
\text { LC }\end{array}$ & 37.5 \\
\hline $\begin{array}{l}\text { Tengah } \\
\text { Sg Batu Anchau } \\
(\mathrm{N} \text { species = } 3)\end{array}$ & Plantation & $\begin{array}{l}\text { Ranidae } \\
\text { Ranidae } \\
\text { Dicroglossidae }\end{array}$ & $\begin{array}{l}\text { Chalcorana raniceps } \\
\text { Pulchrana glandulosa } \\
\text { Fejervarya limnocharis }\end{array}$ & $\begin{array}{l}2 \\
2\end{array}$ & $\begin{array}{l}\mathrm{LC} \\
\mathrm{LC} \\
\mathrm{LC}\end{array}$ & 0 \\
\hline $\begin{array}{l}\text { Sg Batu Anchau } \\
(\mathrm{N} \text { species }=4)\end{array}$ & Plantation & $\begin{array}{l}\text { Dicroglossidae } \\
\text { Rhacophoridae } \\
\text { Ceratobatrachidae } \\
\text { Ranidae }\end{array}$ & $\begin{array}{l}\text { Fejervarya limnocharis } \\
\text { Polypedates leucomystax } \\
\text { Alcalus baluensis } \\
\text { Pulchrana glandulosa }\end{array}$ & $\begin{array}{l}3 \\
2 \\
3 \\
1\end{array}$ & $\begin{array}{l}\mathrm{LC} \\
\mathrm{LC} \\
\mathrm{LC} \\
\mathrm{LC}\end{array}$ & 0 \\
\hline $\begin{array}{l}\text { Ulu } \\
\text { Sg Batu Anchau } \\
(\text { N species = 9) }\end{array}$ & HCVF & $\begin{array}{l}\text { Dicroglossidae } \\
\text { Dicroglossidae } \\
\text { Dicroglossidae } \\
\text { Ranidae } \\
\text { Ranidae } \\
\text { Ceratobatrachidae } \\
\text { Ranidae } \\
\text { Rhacophoridae } \\
\text { Microhylidae }\end{array}$ & $\begin{array}{l}\text { Limnonectes kuhlii } \\
\text { Limnonectes leporinus* } \\
\text { Limnonectes ingeri* } \\
\text { Pulchrana glandulosa } \\
\text { Chalcorana raniceps } \\
\text { Alcalus sariba* } \\
\text { Hylana signata } \\
\text { Nyctixalus pictus } \\
\text { Metaprynella sundana }\end{array}$ & $\begin{array}{r}5 \\
8 \\
10 \\
7 \\
9 \\
1 \\
5 \\
2 \\
2\end{array}$ & $\begin{array}{l}\text { LC } \\
\text { LC } \\
\text { NT } \\
\text { LC } \\
\text { LC } \\
\text { Not listed } \\
\text { LC } \\
\text { NT } \\
\text { LC }\end{array}$ & 33.3 \\
\hline $\begin{array}{l}\text { Sg Linau } \\
(\mathrm{N} \text { species }=7)\end{array}$ & HCVF & $\begin{array}{l}\text { Megophyridae } \\
\text { Microhylidae } \\
\text { Ranidae } \\
\text { Ranidae } \\
\text { Dicroglossidae } \\
\text { Dicroglossidae } \\
\text { Dicroglossidae }\end{array}$ & $\begin{array}{l}\text { Megophyrs nasuta } \\
\text { Metaprynella sundana } \\
\text { Pulchrana signata } \\
\text { Pulchrana glandulosa } \\
\text { Limnonectes paramacrodon } \\
\text { Limnonectes finchi* } \\
\text { Limnonectes leporinus* }\end{array}$ & $\begin{array}{l}4 \\
1 \\
2 \\
2 \\
1 \\
2 \\
1\end{array}$ & $\begin{array}{l}\text { LC } \\
\text { LC } \\
\text { LC } \\
\text { LC } \\
\text { NT } \\
\text { LC } \\
\text { LC }\end{array}$ & 28.6 \\
\hline
\end{tabular}

Note: *Species endemic to Borneo.

$\mathrm{HCV}$ - High Conservation Value.

HCVF - High Conservation Value Forest.

IUCN - International Union for Conservation of Nature's Red List. 
TABLE 5. WATER QUALITY ASSESSMENTS WITH SPECIES ABUNDANCE AT SAREMAS AND SAGERMAS OIL PALM PLANTATIONS

\begin{tabular}{|c|c|c|c|c|c|c|c|c|}
\hline No. & Locality & $\begin{array}{l}\text { Site } \\
\text { cat. }\end{array}$ & $\mathrm{pH}$ & $\begin{array}{l}\text { Turbidity } \\
\text { (NTU) }\end{array}$ & $\begin{array}{l}\text { Salinity } \\
(\mathrm{ppm} / \%)\end{array}$ & $\begin{array}{c}\text { Temperature } \\
\left({ }^{\circ} \mathrm{C}\right)\end{array}$ & $\begin{array}{c}\text { DO } \\
\left(\mathrm{mg} \mathrm{litre}^{-1}\right)\end{array}$ & $\begin{array}{c}\text { No. frog } \\
\text { sp. }\end{array}$ \\
\hline 1 & Hulu Sg Bukit Durang & $\mathrm{HCV}$ & 6.2 & 4.35 & 16.53 & 24.7 & 52.61 & 10 \\
\hline 2 & Hilir Sg Bukit Durang & Plantation & 7.1 & 1.32 & 242.2 & 26.3 & 6.38 & 4 \\
\hline 3 & Sg Kolam & $\mathrm{HCV}$ & 6.8 & 3.64 & 32.24 & 24.9 & 21.9 & 3 \\
\hline 4 & Sg Akuarium & $\mathrm{HCV}$ & 7.7 & 2.74 & 23 & 24.8 & 28.37 & 16 \\
\hline 5 & Hilir Sg Batu Anchau & Plantation & 9.1 & 828.7 & 0.02 & 25.77 & 14.89 & 4 \\
\hline 6 & Tengah Sg Batu Anchau & Plantation & 6.8 & 404 & 58.42 & 28 & 5.82 & 3 \\
\hline 7 & Ulu Sg Batu Anchau & $\mathrm{HCV}$ & 7.2 & 3.49 & 0 & 24.8 & 75.6 & 9 \\
\hline 8 & Sg Linau & $\mathrm{HCV}$ & 6.6 & 5.8 & 0 & 25.2 & 48.3 & 7 \\
\hline
\end{tabular}

Note: HCV - High Conservation Value.

$\mathrm{DO}$ - dissolve oxygen.

NTU - Nephelometric Turbidity Unit.

TABLE 6. MANN-WHITNEY PAIRWISE COMPARISONS OF WATER QUALITY AMONG STUDY SITES

\begin{tabular}{lllllllll}
\hline & A & B & C & D & E & F & G & H \\
\hline A & 0 & 1 & 1 & 1 & 1 & $0.4034^{*}$ & 0.8345 & 0.8345 \\
B & 1 & 0 & 1 & 0.8345 & 0.8345 & 0.5309 & 0.8345 & 0.6761 \\
C & 1 & 1 & 0 & 1 & 1 & $0.4034^{*}$ & 0.6761 & 0.8345 \\
D & 1 & 0.8345 & 1 & 0 & 1 & 0.5309 & 0.7533 & 0.8345 \\
E & 1 & 0.8345 & 1 & 1 & 0 & 0.8345 & 0.5309 & 0.5309 \\
F & $0.4034^{*}$ & 0.5309 & $0.40 .4^{*}$ & 0.5309 & 0.8345 & 0 & $0.4034^{*}$ & $0.2101^{*}$ \\
G & 0.8345 & 0.8345 & 0.6761 & 0.7533 & 0.5309 & 0.4034 & 0 & 0.9166 \\
H & 0.8345 & 0.6761 & 0.8345 & 0.8345 & 0.5309 & $0.2101^{*}$ & 0.9166 & 0 \\
\hline
\end{tabular}

Note: A - Hulu Sg Bukit Durang, B - Hilir Sg Bukit Durang, C - Sg Kolam, D - Sg Akuarium, E - Hilir Sg Batu Anchau, F - Tengah Sg Batu Anchau, G - Ulu Sg Batu Anchau, H - Sg Linau. E, F and B are plantations. A, C, D, G, H are High Conservation Value (HCV) areas. *Significant at $\mathrm{p}<0.5$.

TABLE 7. SPEARMAN'S RS CORRELATION AND ITS SIGNIFICANT VALUE

\begin{tabular}{lllllll}
\hline $\mathbf{0}$ & $\mathbf{1}$ & $\mathbf{2}$ & $\mathbf{3}$ & $\mathbf{4}$ & $\mathbf{5}$ & $\mathbf{6}$ \\
\hline 1 & 0 & 0.84013 & 0.96429 & 0.63234 & 0.53641 & 0.86984 \\
2 & -0.09524 & 0 & 0.43175 & 0.47044 & 0.66458 & $0.37758^{*}$ \\
3 & -0.02395 & -0.32336 & 0 & 0.23249 & $0.03244^{* *}$ & $0.23581^{*}$ \\
4 & 0.2036 & 0.29941 & 0.4759 & 0 & $0.00754^{* * *}$ & $0.034722^{* *}$ \\
5 & -0.2381 & -0.19048 & -0.76648 & -0.87427 & 0 & $0.05119^{* *}$ \\
$\mathrm{~F}$ & 0.072294 & -0.36147 & -0.47274 & -0.76365 & 0.72294 & 0 \\
\hline
\end{tabular}

Note: 1 - pH, 2 - Turbidity (Nephelometric Turbidity Unit), 3 - salinity (ppm/\%), 4 - temperature $\left({ }^{\circ} \mathrm{C}\right)$, 5 - dissolve oxygen (DO) $\left(\mathrm{mg} \mathrm{litre}^{-1}\right), 6$ - No. frog sp. F - significant value.

TABLE 8. KENDALL CORRELATION AND ITS SIGNIFICANT VALUE

\begin{tabular}{lllllll}
\hline $\mathbf{0}$ & $\mathbf{1}$ & \multicolumn{1}{c}{$\mathbf{2}$} & \multicolumn{1}{c}{$\mathbf{3}$} & \multicolumn{1}{c}{$\mathbf{4}$} & \multicolumn{1}{c}{$\mathbf{5}$} & $\mathbf{F}$ \\
\hline 1 & 0 & 0.80457 & 0.89974 & 0.70546 & 0.4579 & 0.79735 \\
2 & -0.0714 & 0 & 0.37782 & 0.37782 & 0.62069 & $0.30437^{*}$ \\
3 & 0.03637 & -0.2546 & 0 & 0.19949 & $0.03221^{* *}$ & $0.36008^{*}$ \\
4 & 0.10911 & 0.25459 & 0.37037 & 0 & $0.00815^{\text {** }}$ & $0.02624^{\text {** }}$ \\
5 & -0.2143 & -0.1429 & -0.6183 & -0.7638 & 0 & $0.07227^{\text {** }}$ \\
F & 0.07413 & -0.2965 & -0.2642 & -0.6416 & 0.51887 & 0 \\
\hline
\end{tabular}

Note: 1 - pH, 2 - Turbidity (Nephelometric Turbidity Unit), 3 - salinity (ppm/\%), 4 - temperature $\left({ }^{\circ} \mathrm{C}\right)$, 5 - dissolve oxygen (DO) (mg litre $\left.{ }^{-1}\right), 6$ - No. frog sp.

F - significant value. 
These kind of microhabitats and heterogeneity was absent in the plantation areas. Additionally, most frogs are also poor dispersers; they never move further than 5 to $10 \mathrm{~km}$ from streams such as the Limnonectes ibanorum, the L. ingeri, the L. kuhlii and the L. leporinus (Inger, 2009).

Disjunctive distributions can occur when suitable habitat is fragmented, which produces fragmented populations, and when that fragmentation becomes so divergent that species movement between suitable habitats to the next is disrupted, isolated population can be produced. Thus, conserving the HCV areas will probably protect those endemic species from becoming vulnerable.

The high turbidity, concentration of salt, and temperature, as well as low DO especially for Tengah Sg Batu Anchau may be due to contamination from the plantation waste or extensive use of insecticides and herbicides. These residues may have contaminated the soil and water; a condition that is highly detrimental to frogs. Studies have shown that the eggs and tadpoles are susceptible to toxic substances, which may not occur in concentrations sufficient to kill adult frogs or even embryos (Blaustein et al., 2003; Broomhall, 2004). Nevertheless, several toxic substances have been found to affect the development of embryos and tadpoles to the point of causing a high percentage of abnormalities or a decrease in the rate of development resulting in prolonged frog-legged periods (Blaustein et al., 2003). For the present study, only one malformed individual (Limnonectes kuhli) was found. There were also observable variations between individuals of Metaprynella sundana in Sg Akuarium with other populations in Borneo such as Matang and Batang Ai National Park. The top of the fingers of these individuals was found to be brighter compared to those of the individuals found elsewhere and this is yet to be explored.

Overall, the HCV areas harboured the highest diversity of species including endemic and near threatened species as compared to the plantation areas. Thus, oil palm plantations should consider setting aside HCV forest and regard these areas as high priority for faunal conservation as also suggested by Norhayati et al. (2014).

\section{CONCLUSION}

As expected the study revealed significant differences in species diversity, species assemblages and physical tolerant (variation in physical factors that an organism population can withstand and continue to thrive in an environment) towards water quality. Thus, it is recommended that the $\mathrm{HCV}$ areas established in any oil palm plantation should be sustainably managed and extended to include a wider forest buffer so that it can harbour endemic and unique species of anurans. For this study, significant quality of HCV areas of Sungai Akuarium, Hulu Sg Batu Anchau and Hulu Sungai Bukit Durang in Wilmar Oil Palm Plantations has been proven and should be given high priority by the management for sustainability of $\mathrm{HCV}$ areas.

\section{ACKNOWLEDGEMENT}

This study was funded by GL (F07)/08/2013/ WILMAR(08). We would like to thank Wilmar Plantations Sdn Bhd and staff as well as Universiti Malaysia Sarawak for the research funding and facilitation throughout the study period.

\section{REFERENCES}

Berry, N J; Phillips Lewis, O L; Simon, L; Hill, J K; Edwards, D P; Tawatao Noel, B; Norhayati, A; David, M; Chey, V K; Maryati, M; Robert, C O and Hamer, K C (2010). The high value of logged tropical forests: Lessons from northern Borneo. Biodiversity Conservation, 19: 985-997.

Blaustein, A R; Romansic, J M; Kiesecker, J M and Hatch, A C (2003). Ultraviolet radiation, toxic chemicals and amphibian population declines Diversity and Distributions. Blackwell Science, Ltd. p. 123-140.

Broomhall, S D (2004). Egg temperature modifies predator avoidance and the effects of the insecticide endosulfan on tadpoles of an Australian frog. J. Applied Ecology, 41: 105-113.

Breuhl, C A and Eltz, T (2010). Fuelling the biodiversity crisis: Species loss of ground-dwelling forest ants in oil palm plantations in Sabah, Malaysia (Borneo). Biodiversity and Conservation, 19: 519-529.

Faruk, A; Belabut, D; Norhayati, A; Knell, R J and Garner, T W F (2013). Effects of oil-palm plantations on diversity of tropical anurans. Conservation Biology, 3: 615-624.

Fayle, T M; Turner, E C; Snaddon, J L; Chey, V K; Chung, A Y; Eggleton, P and Foster, W A (2010). Oil palm expansion into rain forest greatly reduces ant biodiversity in canopy, epiphytes and leaf-litter. Basic and Applied Ecology, 11: 337-345.

Fayle, T M; Turner, E C and Foster, W A (2013). Ant mosaics occur in SE Asian oil palm plantation but not rain forest and are influenced by the presence of nest-sites and non-native species. Ecography, 36: 1051-1057. 
Froster, W A; Snaddon, J L; Turner, E C; Fayle, T M; Cockerill, T D; Ellwood, M D F; Broad, GR; Chung, A Y C; Enggleton, P; Khen, C V and Yusah, K M (2011). Establishing the evidence base for maintaining biodiversity and ecosystem function in the oil palm landscapes of South East Asia. Philosophical Transactions of The Royal Society, 366: 3277-3291.

Gillespie, G R; Ahmad, E; Elahan, B; Evans, A; Ancrenaz, M; Goossens, B and Scroggie, M P (2012). Conservation of amphibians in Borneo: Relative value of secondary tropical forest and non-forest habitats. Biological Conservation, 152: 136-144.

Hammer, O; Harper, D A T and Ryan, P D (2001). PAST: Paleontological statistics software package for education and data analysis. Paleontologia Electronica, 4(1): 9 pp.

Harrison, S and Bruna, E (1999). Habitat fragmentation and large-scale conservation: What do we know for sure? Ecography, 22(3): 225-232.

Inger, R F and Stuebing, R B (2005). A Field Guide to the Frogs of Borneo. $2^{\text {nd }}$ Edition. Natural History Publications. 209 pp.

Inger, R F (2009). Contributions to the natural history of seven species of Bornean frogs. Fieldiana. Zoology Vol. 10: 1552.

Jennings, S; Nussbaum, R; Judd, N and Evans, $\mathrm{T}$ (2003). The High Conservation Value Forest Toolkit. ProForest. http:/ / www.researchgate.net/ publication/228860500, accessed on 15 September 2015.
Kurz, D J; Turner, E C; Aryawan, A A; Barkley, H C; Caliman, J P; Konopik, O P S S and Foster, W A (2016). Replanting reduces frog diversity in oil palm. Biotropica, 48: 487-490. DOI: 10.1111/btp.1232.

Liow, L H; Sodhi, N S and Elmqvist, T (2001). Bee diversity along a disturbance gradient in tropical lowland forests of South East Asia. J. Applied Ecology, 38: 180-192.

Lucey, J M; Tawatao, N; Senior, M J M; Chey, V K; Benedick, S, and Hamer, K C (2014). Tropical forest fragments contribute to species richness in adjacent oil palm plantations. Biological Conservation, 169: 268-276.

Norhayati, A; Ehwan, N and Okuda, T (2014). Assessment of riparian ecosystem on amphibians along a green corridor in oil palm plantation, Pasoh, Negeri Sembilan, Peninsular Malaysia. Sains Malaysiana, 43(5): 655-666.

Sanath, K (2008). HCV AREAS scoping assessment of Saremas 1\&2, Sagermas and Kaminsky Estate of PPB Oil Palms Berhad in Sarawak. 23 pp.

Sheridan, J A; Howard S D; Yambun, P; Rice, J L; Cadwalladerstaub, R; Karoulus, A and Bickford, D (2012). Novel behaviors of Southeast Asian rhacophorid frogs (Anura: Rhacophoridae) with an updated anuran species list for Danum Valley, Sabah, Malaysian Borneo. Tropical Natural History, 12: $1-8$.

Wu, Z; Li, Y and Murray, B R (2006). Insular shifts in body size of rice frogs in the Zhou shan archipelago, China. J. Animal Ecology, 75: 1071-1080. 


\section{SELECTED ANURAN SPECIES AT SAREMAS AND SAGERMAS OIL PALM PLANTATIONS}

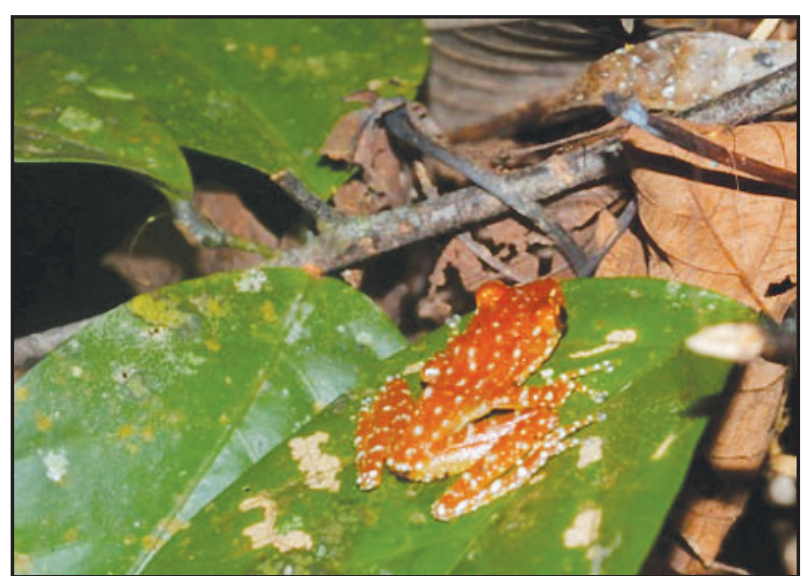

Nyxtixalus pictus

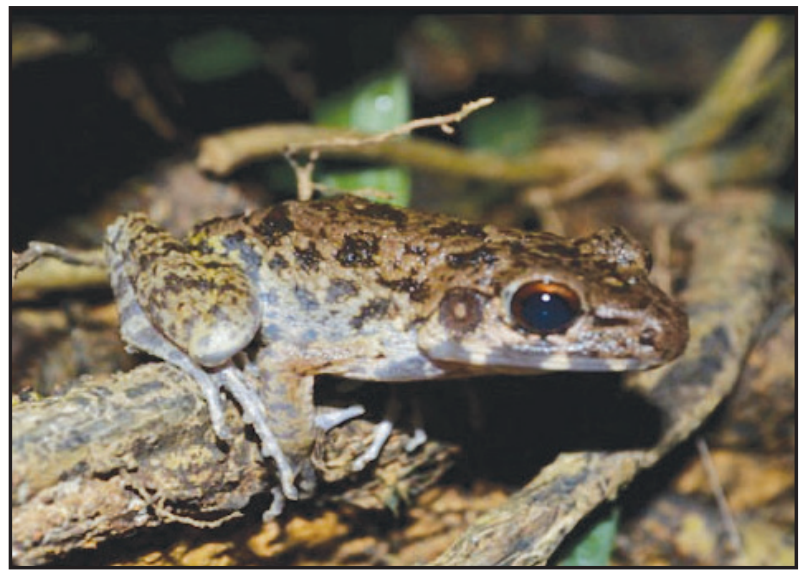

Pulchrana glandulosa

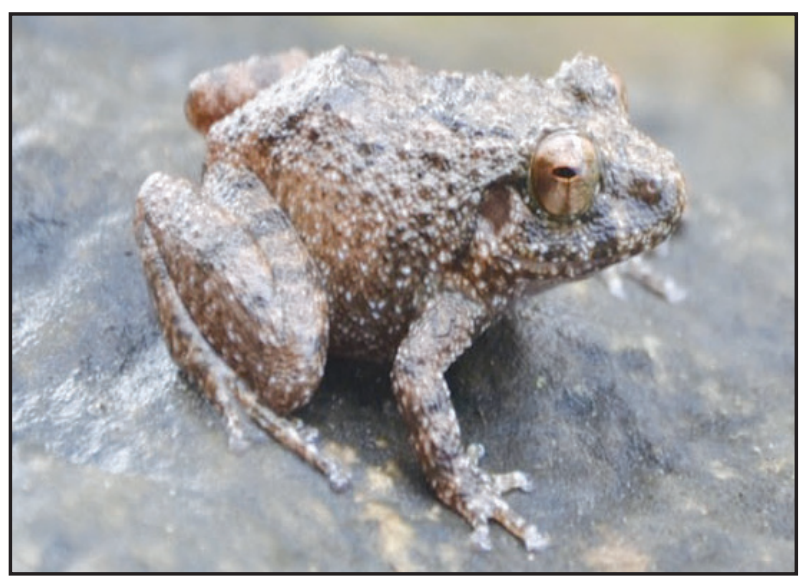

Alcalus sariba

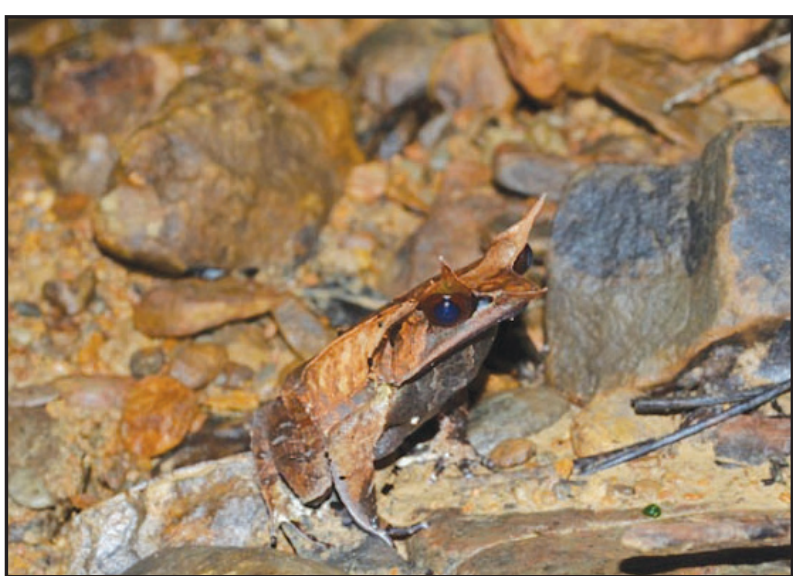

Megophyrs nasuta

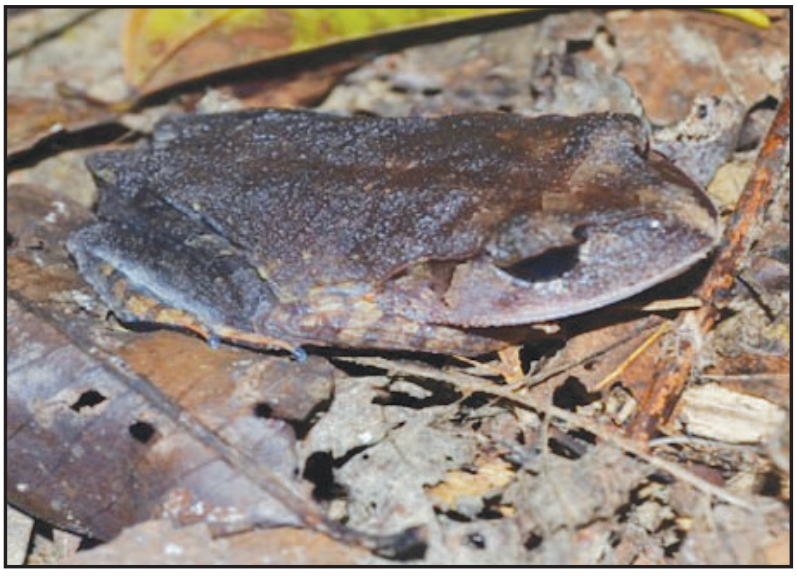

Leptobrachium abbotti

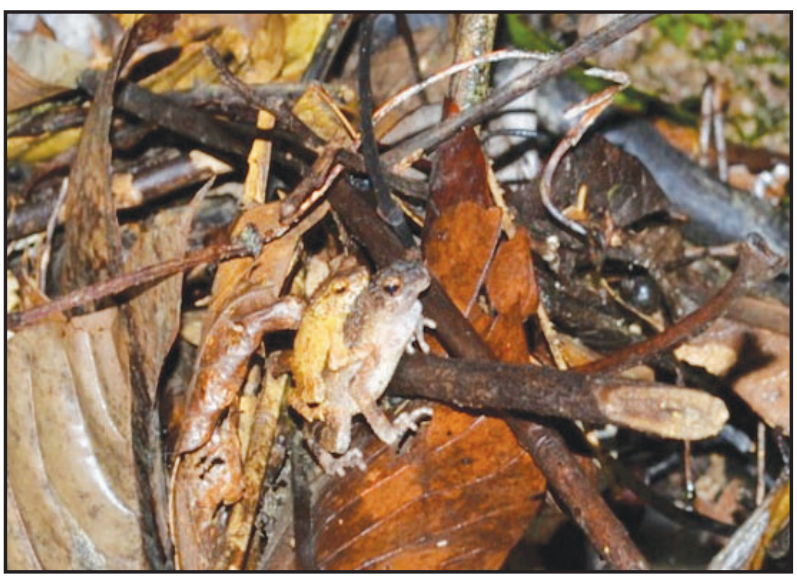

Ingerophrynus divergens in amplexus 